\title{
Optimal Conditions for Clostridium difficile Toxins Production
}

\author{
Medhat M. Taha* \\ Veterinary Serum and Vaccine Research Institute, Agriculture Research Center (ARC), \\ Abbasia, Cairo, Egypt \\ *Corresponding author
}

\section{A B S T R A C T}

\begin{tabular}{|l|}
\hline K e y w o r d s \\
$\begin{array}{l}\text { Clostridium } \\
\text { difficile, } \\
\text { Toxins }\end{array}$ \\
\hline Article Info \\
\hline $\begin{array}{l}\text { Accepted: } \\
\text { 26 September 2020 } \\
\text { Available Online: } \\
10 \text { October } 2020\end{array}$ \\
\hline
\end{tabular}

Clostridium difficile causes acute enterocolitis among different animal species. The disease is mediated primarily through two exotoxins known as TcdA and TcdB, their synthesis depends mainly on growth of the organism which is controlled by many factors. Variations in the production of these toxins are affected by the medium and its nutrients, the using of carbon source in toxin production medium will affect the toxin level. In this study, we use different sources of carbon in the toxin production media, It was found that glucose and fructose gave low toxin production levels while using $1 \%$ starch in toxin production media for 5 days incubation and then separation of the produced toxins using $0.22 \mathrm{um}$ Millipore filter, high level of Clostridium difficile toxins obtained that could be used in the future for preparation of formalized toxoid against enterocollitis from locally isolated strain of Clostridium difficile .

\section{Introduction}

Clostridium difficile is a gram-positive, anaerobic bacterium that can be found in many animal species. It causes $C$. difficile infection (CDI) which is an important disease in humans. Its role as an animal pathogen ranges from important to unclear to apparently irrelevant, depending on the animal species. The transmission of this organism occurs by the fecal oral route and the most important risk factor is the antibiotic therapy $(1,2)$.

Isolates of Clostridium difficile mostly produce two major virulence factors that causes damage the epithelium of the colon, toxin A enterotoxin (TcdA) and toxin B cytotoxin (TcdB). Clostridium difficile toxin A (308KDa) is known to cause apoptosis of the epithelium cells of colon, which consequently lead to colitis (3).

On the other side, toxin B (270KDa) acts synergistically with toxin A as a cytotoxin only after the epithelium has been injured by toxin A (4). TcdB doesn't cause fluid accumulation and it is more cytotoxic to cultured cell lines than TcdA, less than a picogram of cytotoxin $B$ causes rounding up of the cells, and death of cells. Hence, both these toxins are targets for laboratory diagnostic tests and for vaccine development $(5,6)$. 
This study was planned to reach the requirements essential in toxin production media for synthesis of toxins including source of carbons and its concentration, incubation period and filtration effect, that consequently produce high yield of toxins A and B from locally isolated Clostridium difficile strain to be used in vaccine production.

\section{Materials and Methods}

\section{Strain}

Locally isolated strain of Clostridium difficile from facecal samples and rectal swab of foals from EAO El-Zahraa Stud., was kindly obtained from Anaerobic Dept., Serum and Vaccine Research Inst., Cairo. (7)

\section{Bacterial growth}

Previously isolated Clostridium difficile was cultivated on the specific agar base (CM0601, Oxoid LTD, England) with Clostridium difficile selective supplement (SR0096E, Oxoid LTD, England) and incubated for 48 hrs anaerobically (8).

\section{Toxins production}

To reach the most effective factors for high toxins production from Clostridium difficile culture, the bacterial culture was cultivated in the two toxin production media under test (thioglycollate and peptone media in concentration $29 \mathrm{mg} / \mathrm{L} 32 \mathrm{gm} / \mathrm{L}$ respectively) (7). $0.2 \%$ Magnesium sulphate and $0.5 \%$ sodium chloride were added for each to improve cell growth and toxin production (9, 20). Different type of sugars (glucose, fructose, lactose, sucrose, starch and dextrin) added and the culture incubated for $24 \mathrm{hrs}, 72$ hrs. and 5 days separately at $37{ }^{\circ} \mathrm{C}$ then ELISA was done to determine the most effective sugar on toxin production according to the sufficient incubation period for toxin production and the the toxins productivity was further tested by different concentrations of the used source of carbon $(0.5 \%, 1 \%$ and $1.5 \%)$. After determining the most effective toxin production medium with effective source of carbon and its concentration as well as the incubation time, Clostridium difficile culture was cultivated on the media with its resulted optimal conditions and then separated using Millipore filtration system (using Pellicon® 2 Cassette Filter Module GVPP $0.22 \mu \mathrm{m}$ ) and the toxin productivity was determined using ELISA.

\section{ELISA}

Toxins $\mathrm{A}$ and $\mathrm{B}$ of Clostridium difficile can be specifically determined using RIDASCREEN® Clostridium difficile Toxin A/B kit (R-Biopharm AG, Dermstadt, Germany), which is an immune assay depends on monoclonal antibodies of toxins $\mathrm{A}$ and $\mathrm{B}$ in sandwich type method, these monoclonal antibodies are attached to the well surface of the microwell plate. The prepared toxin (from each medium) and the positive and negative controls were placed into the wells of microplate with the biotinylated antitoxin $\mathrm{A} / \mathrm{B}$ antibodies as a conjugate 1 . then the plate incubated for $60 \mathrm{~min}$ and the wells washed using the washing buffer (phosphate buffered $\mathrm{NaCl}$ solution), and the streptavidin polyperoxidase (Conjugate 2) was added in each well then the plate reincubated for 30 min and washed for 3 times and the substrate (Hydrogen peroxide/TMB) was added and finally the reaction stopped using $1 \mathrm{~N}$ sulpheric acid after $15 \mathrm{~min}$. the plate was read at $450 \mathrm{~nm}$.

\section{Results and Discussion}

Clostridium difficile can cause clinical conditions ranging from mild diarrhea to fulminant disease. Approximately $30 \%$ of infected animals with $C$. difficile infection 
(CDI) will progress to severe disease (10). Mainly, the symptoms of CDI related to the production of two exotoxins namely, toxin A (TcdA) and toxin B (TcdB) (11). TcdA and $\mathrm{TcdB}$ are 308 and $270 \mathrm{kDa}$ proteins, respectively. The toxins belong to the family of large clostridial toxins (LCTs).

Clostridium difficile cells grown in rich media typically express TcdA and TcdB during the stationary phase (12). The toxin expression has been reported to be affected by environmental stimuli including the presence of a rapidly metabolizable carbon source. The genes encoding TcdA (tcdA) and TcdB (tcdB) are located within a $19.6-\mathrm{kb}$ chromosomal region, the pathogenicity locus (PaLoc). (13).

Non-toxigenic Clostridium difficile strains, however, can acquire the PaLoc from toxigenic strains through horizontal gene transfer, resulting in the conversion of nontoxigenic strains to toxin producers (14). Also, the PaLoc in most pathogenic strains encodes three proteins, TcdR, TcdC and TcdE, which regulate the toxin production and secretion. TcdR is a member of the extracytoplasmic function family of alternative sigma factors and plays a critical role in activating the expression of $t c d A$ and $t c d B$. Additionally, TcdR positively regulates its own expression, while TcdC act as an antisigma factor that negatively regulates toxin expression, TcdE acts as a holing like protein to facilitate the release of Clostridium difficile toxins to extracellular environment (15).

For instance, when cells are grown in rich medium, the toxin genes are transcribed only when the cells enter stationary phase, i.e., when some form of nutrient limitation or accumulation of growth inhibiting substances occurs. Once expression of the toxin genes is induced, the toxin proteins accumulate inside the cell and are slowly released over the course of several hours (16).
Sugar is the generic name of soluble carbohydrates. Simple sugars, also called monosaccharides, include glucose and fructose,. Disaccharides refereed to are molecules composed of two monosaccharides joined by a glycosidic bond. as sucrose (glucose and fructose) and lactose (galactose and glucose). Polysaccharides are longer chains of monosaccharides as starch (which is a carbohydrate consists of large number of glucose units linked by glycosidic bonds and dextrin a mixture of polymaers of D-glucose units linked by $\alpha-(1 \rightarrow 4)$ or $\alpha-(1 \rightarrow 6)$ glycosidic bonds).

Previously (14), Clostridium difficile colonies were harvested on Peptone, Thioglycollate broth, cooked meat medium and brain heart infusion medium and on same media with addition of $1 \%$ glucose for each, then incubated 72 hrs. anaerobically. Then, ELISA was carried out to determine the most medium that could be used for toxin production. It was found that thioglycolate medium with addition of $1 \%$ glucose or peptone with $1 \%$ glucose showing best results for toxin production of Clostridium difficile.

In this study we study the effect of presence of different types of carbohydrates as source of carbon with concentration $1 \%$ on thioglycollate and peptone media, and taking samples after 1 day, 3 days and 5days incubation, then the samples were examined by ELISA. as showed in table 1. From table 1, we can conclude that at day 1 in all samples the toxin level not reach satisfactory level while in day 3 the level of the toxin increases as well as in day 5, this completely agreed with (18) as they said that in the early exponential phase, a high level of tcdC and low levels of tcdA,B,D,E transcripts were detectable; this was inverted in the stationary phase, suggesting that TcdC might have a negative influence on transcription of the other genes. 
Table.1 Effect of different sources of carbon on Clostridium difficile toxin production as determined by ELISA

\begin{tabular}{|c|c|c|c|c|c|c|c|}
\hline \multicolumn{2}{|c|}{ Medium } & \multicolumn{3}{c|}{ Thioglycollate } & \multicolumn{3}{c|}{ Peptone } \\
\hline $\begin{array}{c}\text { Incubation time } \\
\text { Type of carbohydrates } \mathbf{1 \%})\end{array}$ & $\mathbf{1}$ day & $\mathbf{3}$ days & $\mathbf{5}$ days & $\mathbf{1}$ day & $\mathbf{3}$ days & $\mathbf{5}$ days \\
\hline Monosaccharide & glucose & $\mathbf{0 . 1 9 0}$ & $\mathbf{0 . 4 1 0}$ & $\mathbf{0 . 4 8 9}$ & $\mathbf{0 . 2 1 5}$ & $\mathbf{0 . 4 9 6}$ & $\mathbf{0 . 6 3 2}$ \\
\hline & fructose & $\mathbf{0 . 1 7 5}$ & $\mathbf{0 . 3 2 0}$ & $\mathbf{0 . 4 5 5}$ & $\mathbf{0 . 2 2 3}$ & $\mathbf{0 . 4 7 2}$ & $\mathbf{0 . 5 2 3}$ \\
\hline \multirow{2}{*}{ Disaccharides } & sucrose & $\mathbf{0 . 2 2 0}$ & $\mathbf{0 . 5 4 5}$ & $\mathbf{0 . 7 1 0}$ & $\mathbf{0 . 2 0 6}$ & $\mathbf{0 . 6 2 3}$ & $\mathbf{0 . 7 2 0}$ \\
\hline & Lactose & $\mathbf{0 . 2 7 2}$ & $\mathbf{0 . 5 1 0}$ & $\mathbf{0 . 8 1 4}$ & $\mathbf{0 . 1 9 3}$ & $\mathbf{0 . 6 1 0}$ & $\mathbf{0 . 8 0 9}$ \\
\hline \multirow{2}{*}{ Polysaccharides } & starch & $\mathbf{0 . 2 9 0}$ & $\mathbf{0 . 7 9 2}$ & $\mathbf{1 . 0 1 9}$ & $\mathbf{0 . 2 0 3}$ & $\mathbf{0 . 9 4 4}$ & $\mathbf{1 . 0 5 7}$ \\
\hline & dextrin & $\mathbf{0 . 3 0 6}$ & $\mathbf{0 . 7 2 0}$ & $\mathbf{0 . 9 2 3}$ & $\mathbf{0 . 2 2 0}$ & $\mathbf{0 . 8 3 3}$ & $\mathbf{0 . 9 1 2}$ \\
\hline
\end{tabular}

Positive control $: 1.232$, negative control : 0.031 and cut off : 0.181

Table.2 Effect of different concentrations of starch on toxin production as determined by ELISA

\begin{tabular}{|c|c|c|c|}
\hline Starch conc. \% & 0.5 & 1 & 1.5 \\
\hline Thioglycollate & $\mathbf{0 . 8 1 0}$ & $\mathbf{0 . 9 7 5}$ & $\mathbf{0 . 9 9 3}$ \\
\hline Peptone & $\mathbf{0 . 8 2 3}$ & 1.092 & $\mathbf{1 . 0 7 1}$ \\
\hline
\end{tabular}

Positive control: 1.270 , negative control: 0.043 and cut off: 0.193

Table.3 O.D. for separated toxin in different media

\begin{tabular}{|c|c|c|}
\hline & Peptone medium & Thioglycollate medium \\
\hline Whole culture & 1.092 & $\mathbf{0 . 9 7 5}$ \\
\hline Separated toxin & 1.179 & 1.132 \\
\hline
\end{tabular}

Positive control:1.270, negative control:0.043 and cut off:0.193

Starch and dextrin when used as sources of carbon in toxin production media of Clostridium difficile, high toxin levels obtained specially with starch in comparing to the other carbohydrates used. Also it was cleared that glucose and fructose showing low results in comparing with other sources of carbon, that returned to the fact that $C$. difficile, toxin production increases as cells enter the stationary phase and is modulated in response to different environmental signals and the transcription of $\operatorname{tcd} A$ and $\operatorname{tcdB}$ is repressed by the presence of rapidly metabolizable carbohydrates, such as glucose or other sugars taken up by the phosphoenolpyruvate-dependent phosphotransferase system (PTS) as fructose (19).while using sucrose and lactose gave moderate levels of toxin production.

From the obtained results, it could be concluded that starch is the best source of carbon for Clostridium difficile toxin production media for 5 days incubation.

Moreover, different concentrations of starch $(0.5 \%, 1 \%$ and $1.5 \%)$ studied with thioglycollate and peptone media, as showed in table 2.

From table 2, it was concluded that using $0.5 \%$ starch in thioglycollate or peptone 
media gave low toxin level in comparing to $1 \%$ and $1.5 \%$ concentrations, while $1 \%$ and $1.5 \%$ show approximately near results, thus economically $1 \%$ of starch could be used.

Table 3 showed that using separated toxin gave high OD readings than that of whole culture of Clostridium difficile using either peptone or thioglycollate media. This was completely agreed with (20) who used cultured filtrate to produce Clostridium difficile toxoid vaccine.

Finally, it could be concluded that for reaching high toxin levels for Clostridium difficile, toxin production medium should constitute of Peptone or thioglycollate media with $1 \%$ starch, $0.2 \%$ magnesium sulphate and $0.5 \%$ sodium chloride for 5 days incubation, then filtrated using $0.22 \mathrm{um}$ sized pores and that consequently could be used to prepare formalized toxoid vaccine against eneterocolitis disease to prevent the economic losses caused by Clostridium difficile

\section{References}

Antunes A, Camiade E and Monot M (2012) Global transcriptional control by glucose and carbon regulator CcpA in Clostridium difficile. Nucleic Acids Res 40:10701-18.

Awad MM, Johanesen PA and Carter GP (2014) Clostridium difficile virulence factors: Insights into an anaerobic spore-forming pathogen. Gut Microbes 5:579-93.

Brouwer MS, Roberts AP, Hussain H (2013) Horizontal gene transfer converts nontoxigenic Clostridium difficile strains into toxin producers. Nat. Commun 4:2601-2606.

Cato EP, George WL and Finegold SM (1986) Genus Clostridium Prazmowski 1880, 23 In :Bergy`s Manual os Systematic Bacteriology, volume 2: 1141-1142.

Clostridium difficile infection :review European Journal of Clinical Microbiology and Infectious Disease 38: 1211-1221.

Cussó, L., E. Reigadas , P. Muñoz, Manuel Desco and E. Bouza (2020) Evaluation of Clostridium difficile Infection with PET/CT Imaging in a Mouse Model. Mol Imaging Biol Jun; 22(3): 587-592.

Czepiel J., Miroslaw D., Hanna P. ,Kuijper Ed, Perucki W., Mielimonka A., Garlilick A. and Biesiada G. (2019)

Darkoh C, DuPont HL and Norris SJ (2015) Toxin synthesis by Clostridium difficile is regulated through quorum signaling. mBio, 6:e02569

Dupuy B, and Sonenshein AL. (1998) Regulated transcription of Clostridium difficile toxin genes. Molecular microbiology. 27:107-20.

Eric M. Ransom1, Gabriela M. Kaus1, Phuong M. Tran, Craig D. Ellermeier, and David S. Weiss (2018) Multiple factors contribute to bimodal toxin gene expression in Clostridioides (Clostridium) difficile. Mol Microbiol. 110(4): 533-549

Fathi Najafi, M., Hemmaty, M., Navidmehr, J., Afsharian, M., Farhoodi, M. and Zibaee, S. (2020) Improvement in the Growth and $\alpha$-toxin Production of Clostridium septicum by Magnesium Sulfate. Archives of Razi Institute, Vol. 75, No. 2 (2020) 219-225

Hafiz S and Oakely CL (1976) Clostridium difficile, Isolation and characteristics. Journal of Medical Microbiology, 9: 129-136.

Karasawa T, Maegawa T and Nojiri T (1997) Effect of arginine on toxin production by Clostridium difficile in defined medium. Microbiol. Immunol 41:5815.

Keel M. K. and Songer J.G. (2006) The 
comparative pathology of Clostridium difficile associated disease. Vet.Pathol. 43:223-2210.

Maja Rupnik and Sandra Janezica (2016) An Update on Clostridium difficile Toxinotyping. Journal of Clincal Microbiology 54(1): 13-18.

Mitchell Tj. Ketley J.M. and Haslam S.C (1986) Effect of toxin A and B of Clostridium difficile on rabbit ileum and colon. GUT 27: 78-85.

Samer Aboudola, Karen L. Kotloff, Lorraine Kyne, Michel Warny, Eoin C. Kelly, Stavros Sougioultzis, Paul J. Giannasca, Thomas P. Monath, and Ciara'n P. Kelly (2003) Clostridium difficile Vaccine and Serum Immunoglobulin G Antibody Response to Toxin A. Inf. and Imm., 71(3): 1608-1610 .

Segner, W. P., C. F. Schimdt, AND J. K. Boltz (1971) Minimal Growth Temperature, Sodium Chloride Tolerance, $\mathrm{pH}$ Sensitivity, and Toxin
Production of Marine and Terrestrial Strains of Clostridium botulinum Type C App. Microbiol., 1025-1029.

Taha MM (2014). Isolation and identification of Clostridium difficile from horses. Zag Vet J, 42: 124-130.

Taha MM (2019) Studies on Virulence Factors of Locally Isolated Clostridium difficile from Horses Inter J Vet Sci, 8(1): 39-42.

Thomas H. , Veit B. , Manfred W., Petra L. , Markus S. and Christoph V. (1997) Transcription analysis of the genes $t c d A-E$ of the pathogenicity locus of Clostridium difficile . Eur. J. Biochem. 244, 735-742

Young Ha Kim and Ho Kim (2019)

Clostridium difficile Toxin A Upregulates Bak Expression Through PGE2 Pathway in Human Colonocytes. J. Microbiol Biotechnol 28:29(10):1675-1681

\section{How to cite this article:}

Medhat M. Taha. 2020. Optimal Conditions for Clostridium difficile Toxins Production. Int.J.Curr.Microbiol.App.Sci. 9(10): 3469-3474. doi: https://doi.org/10.20546/ijcmas.2020.910.400 